\title{
Breast miofibroblastoma in postmenopausal women: case report
}

\section{Summary}

Myofibroblastoma is a rare benign mesenchymal tumor of the breast. This tumor has been previously described in the pathological and surgical literature, but there are limited reports on the women. It tends to be more prevalent in the male breast.

As spindle cell lesions of the breasts are rare, when encountered on core biopsy, it can be challenging to differentiate between benign and malignant lesions. Therefore, surgical excision may be necessary. In the clinical practice, mammary myofibroblastoma tends to present as a unilateral, firm, mobile, painless tumor that may demonstrate slow, steady growth over months to years.

Most myofibroblastomas are immunoreactive for CD34, actin, CD10, and desmin, and also usually express estrogen receptor. We show a female breast myofibroblatoma in a Latin American patient, with a difficult diagnosis. The final pathological report was made with immunohistochemistry. Clinical, radiological features are non-specific, even eosin-hematoxylin dye diagnosis needs support by immunohistochemistry.

The majority of the myofibroblastomas are immunoreactive for CD34, actin, CD10, and desmin. They also usually express estrogen receptor (ER) and progesterone receptor (PR), while variable expressing androgen receptor (AR). They are not immunoreactive for cytokeratins, EMA, S100, HMB-45, and CD117. Pathological definitive diagnosis always must be, because this tumor is frequently misdiagnosis like a benign tumor, metastatic tumor, sarcoma or epithelial malignant tumor.

Keywords: myofibroblastoma, breast rare tumor, immunohistochemical breast tumor
Volume 10 Issue 4 - 2019

\author{
Victor EValdespino,' Israel Lopez \\ Matamoros, ${ }^{2}$ Paola X Ferrer, ${ }^{2}$ Antonio Garcia \\ Rodriguez, ${ }^{3}$ Claudia Meixueiro Calderon, ${ }^{4}$ \\ Victor Valdespino Gomez \\ 'Oncology gynecology Gynecological and Obstetrician Hospital \\ 4 IMSS, Mexico \\ Junior Doctor oncology gynecology, Gynecologycal and \\ Obstetrician Hospital 4, Mexico \\ ${ }^{3}$ Pathology Gynecological and Obstetrician Hospital, Mexico \\ ${ }^{4}$ IMSS, Pathology Navy Medical Center, Mexico \\ ${ }^{5}$ Oncology Surgeon, Gynecologycal and Obstetrician Hospital \\ 4, Mexico
}

\section{Correspondence: Victor E.Valdespino, Social Security Mexican Institute. Gynecologycal and Obstetrician Hospital 4, Mexico City, Navy Medical Center, Mexico City, Mexico, Tel +525573678619, Email edvaldspinocg@yahoo.com}

Received: June 23, 2019 | Published: July 22, 2019

\section{Introduction}

Benign mesenchymal breast entities may mimic invasive carcinoma on imaging and morphology, and their diagnoses can therefore, be challenging for clinicians, radiologists, and pathologists.

Due to the broad morphologic spectrum of miofibroblastoma, this uncommon benign tumor may mimic a wide variety of both benign and malignant breast spindle cell lesions, causing a potential diagnostic pitfall. It is composed of tumor cells derived from Schwann cells. ${ }^{1}$

Pathologists have to pay close attention to the differential diagnosis of these lesions and to recognize the benign nature of these entities, further preventing diagnostic mistakes and unnecessary treatment.

Myofibroblastoma of the breast is a rare benign tumor of mesenchymal origin composed predominantly of myofibroblasts. It was first described in 1987 by Wargotz. ${ }^{3}$

Myofibroblastoma of the breast, presents clinically as a solitary, painless, slow growing, circumscribed, mobile mass.

Features to aid in recognition of the lesion, include absent to rare mitotic activity and the dense collagenised stroma. Diagnostic difficulties arise, particularly in core needle biopsies, when myofibroblastomas demonstrate atypical or several features. ${ }^{4}$

Differential diagnosis of miofibroblastoma includes other pure mesenchymal lesions such as fibromatosis, nodular fasciitis, neurilemmoma, stromal sarcoma, spindle cell lipoma, spindle-cell carcinoma, phyllodes tumor, cellular angiolipoma, nerve sheath tumors, and invasive lobular carcinoma. ${ }^{5,6}$
Surgical excision should be necessary for precise diagnostic and treatment. ${ }^{7}$ We present a case of myofibroblastoma in the female patient with 60 years old.

Miofibroblastoma runs an indolent clinical course. Treatment by excision is recommended and curative in this etiologies. No adjuvant therapy is needed. These lesions have been reported to mimic carcinoma.

\section{Case report}

The patient is 60years old, female, Latin American phenotype, 2 gestation, 2 child-birth, hypertension since 15 years ago, with family history on cancer, testicular cancer in his father, sought out ambulatory care due to a palpable nodule of slow growth in her left breast, which had appeared approximately 6 months before. On physical examination, the patient had large breasts and a palpable nodule of approximately $3 \mathrm{~cm}$ in diameter (Figure 1), of fibroelastic consistency, located in her left breast's lateral-superior quadrant, with smooth and regular borders, movable and nonpainful to palpation, free axillary lymph nodes and absence of any discharge. Mammography, ultrasonography and core biopsy of the nodule were performed. Upon return, the mammogram presented an oval image in the left breast, with sharp edges, BIRADS $4 \mathrm{C}$ was a grade of mammography (Figure 2). Ultrasound revealed a nodular, hypoechoic image with lobulated contours, with a near to, cutaneous plane, measuring approximately $34 \times 25 \times 27 \mathrm{~mm}$. The report, of core biopsy, it was a fusocellular neoplasm with epitheloid areas probably phyllodes tumor or metaplastic carcinoma. Biopsy of the nodule was performed with the anatomopathological examination, immunohistochemistry was requested, vimentin positive, desmine 
positive, CD34 positive, smooth muscle positive, estrogen positive, this suggested sarcomatoid carcinoma.

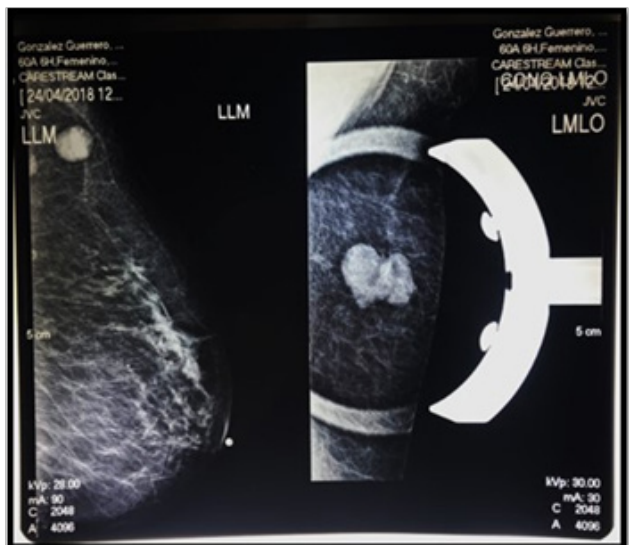

Figure I The patient had large breasts and a palpable nodule of approximately $3 \mathrm{~cm}$ in diameter.

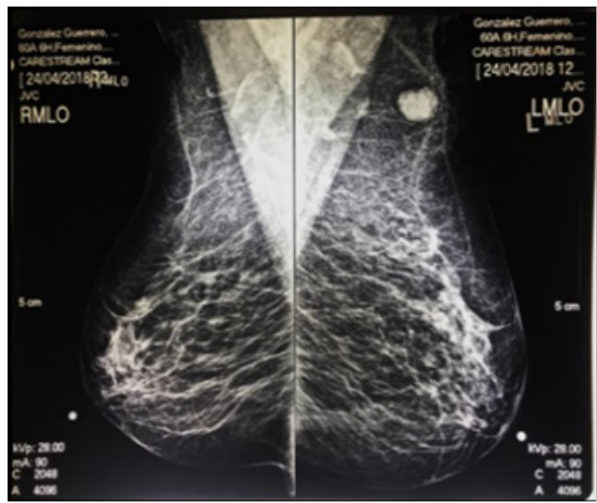

Figure 2 The mammogram presented an oval image in the left breast, with sharp edges, BIRADS $4 C$ was a grade of mammography.

Quadrantectomy was performed as surgical treatment, with the removal of the tumor. All the material was sent for anatomopathological study.

Macroscopically report of the tumor was a nodular lesion, with well delimited, elastic consistency, surrounded by fat tissue. Also, we performed a radical axillary dissection with 17 nodes metastasis-free (Figures 3-8).

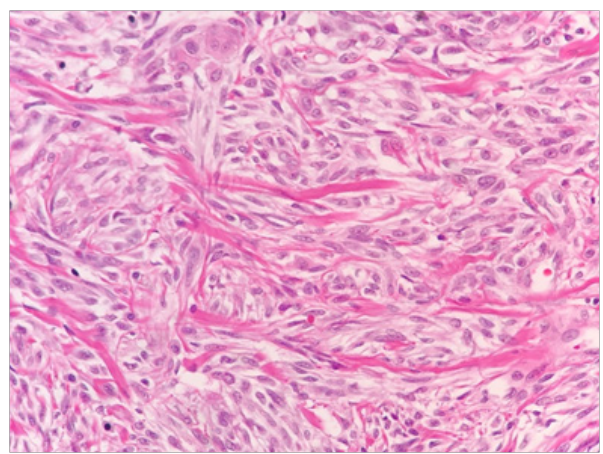

Figure 3 Hematoxilin and Eosin dye show us mesenchymal neoplasia, constituted it by fusiform cells, ovoid nucleus, no cellular atypia, arranged it in the layer, with hyalinized collagen, with no mitosis activity.

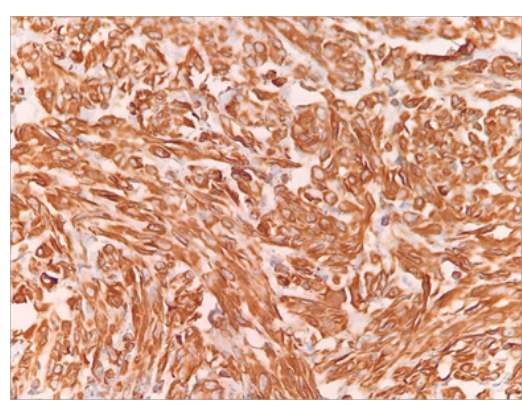

Figure 4 The cells show us strong positivity to Vimentin.

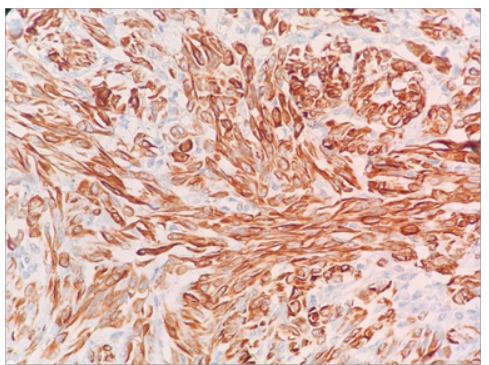

Figure 5 Desmin positive.

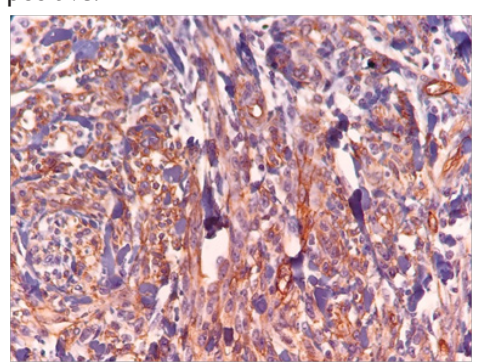

Figure 6 CD34 positive.

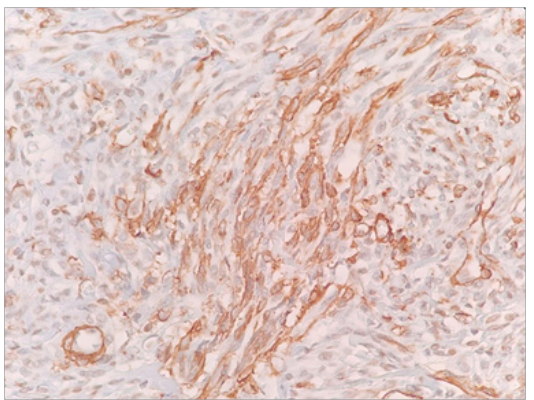

Figure 7 Actin smooth muscle positive.

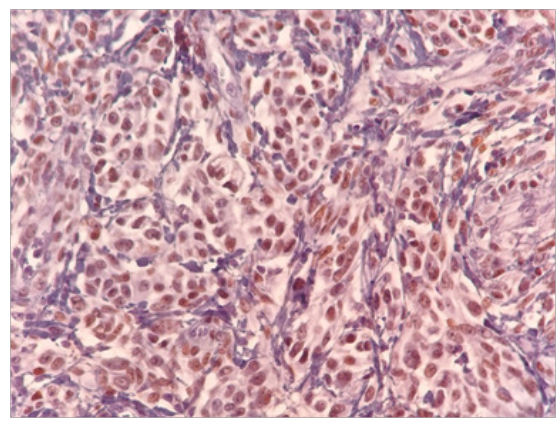

Figure 8 Estrogen positive. 
The final diagnosis was classic breast myofibroastoma. We performed axillary lymphadenectomy by carcinoma suspect.

\section{Discussion}

Differential diagnosis should be made, among others, with, carcinoma, sarcoma and metastases, and benign tumors. ${ }^{8}$

There are variants of myofibroblastoma which have been identified histopathologically. These are classic, cellular, collagenous, lipomatous, infiltrative, myxoid, epithelioid, and decidua-like variants which have been identified. ${ }^{9}$

Immunohistochemistry plays a fundamental role in the diagnosis of these tumors.

In this report, we confirmed positivity for the antibodies, desmin, and actin, CD 34, smooth muscle, estrogen, vimentin, revealing myofibroblastoma through morphological and immunohistochemical findings. ${ }^{10,11}$

Genetically, myofibroblastoma has been shown to exhibit deletion at the chromosome $13 \mathrm{q}$ region, with most cases exhibiting $13 \mathrm{q} 14$ deletion, similar to other benign mesenchymal and stromal neoplasms including spindle cell lipoma and cellular angiofibroma. ${ }^{12,13}$

Tumor's surgical resection is the treatment of choice, and so far there are no descriptions of local recurrences or metastases of myofibroblastoma. ${ }^{14}$ Is not necessary nodal biopsy or nodal whole dissection, but transoperative pathological diagnosis could report carcinoma or sarcomatoid malignant tumor, and practice a surgical treatment on lymph nodes; like this report.

Cytologically, myofibroblastic breast tumor is characterized by the presence of abundant, randomly arranged single and clustered benign spindle-shaped mesenchymal cells with scant cytoplasm, elongated or oval-shaped nuclei and a variable degree of cellularity. The most cellular regions seem to have a hypoechoic appearance on ultrasonography, and an ultrasound-guided core biopsy of those regions allows a precise diagnosis. Ower group performed a core biopsy of the breast tumors but some times is necessary a lumpectomy or wide resection get a price diagnosis.

Benign mesenchymal tumors of the breast are rare and exhibit histological features that can mimic malignant breast lesions and miss diagnosis this kind of tumors. ${ }^{15}$

There are variants of myofibroblastoma which have been identified histopathologically. These are classic, cellular, collagenous/fibrous, lipomatous, infiltrative, myxoid, epithelioid, and decidua-like variants which have been identified. ${ }^{16}$

Although myofibroblastomas usually display benign features upon imaging, most of the masses are treated with surgical excision. The prognosis of myofibroblastoma is very good with no local tumor recurrences or distant metastasis. ${ }^{17}$

Lee and coworkers reported a myofibroblastoma in male but they used, mammographic studies, ultrasound, magnetic resonance, and computed tomography; but the ower group does not use because, those elements (magnetic resonance and computed tomography) increased the cost of medical attention, and we have limited access to this resource. ${ }^{18}$

\section{Conclusion}

Our cases illustrate miofibroblastoma are relatively rare breast neoplasms, they should always be considered in the differential diagnosis of benign and malignant lesions. Since clinically and radiologically these lesions can mimic invasive carcinoma and the definitive diagnosis is made by pathology, pathologists should bear this in mind to avoid misdiagnosing breast carcinoma, which could lead to unnecessary surgery, and treatments.

Myofibroblastoma is a rare tumor characterized by spindle-shaped mesenchymal cells derived from fibroblasts. Myofibroblastoma of the breast is an extremely rare benign tumor, which might be confused with malignancy, clinically and radiologically and pathological. We have to keep in mind this unfrequent tumor and others when we evaluate a breast tumor.

\section{Acknowledgments}

None.

\section{Funding}

None.

\section{Conflicts of interest}

Authors disclose no conflict of interests in publication of this study.

\section{References}

1. Simoes CC, Qiu S, Nguyen QD, et al. Rare mesenchymal breast entities that mimic malignancy: a single-institution experience of a challenging diagnosis. Cureus. 2019;11(3):e4000.

2. Magro G. Mammary myofibroblastoma: a tumor with a wide morphologic spectrum. Arch Pathol Lab Med. 2008;132(11):813-820.

3. Wargotz ES, Weiss SW, Norris HJ. Myofibroblastoma of the breast. Sixteen cases of a distinctive benign mesenchymal tumor. Am J Surg Pathol. 1987;11(7):493-502.

4. Torous VF, Schnitt SJ, Collins LC. Benign breast lesions that mimic malignancy. Pathology. 2017;49(2):181-196.

5. Magro G. Mammary myofibroblastoma: a tumor with a wide morphologic spectrum. Arch Pathol Lab Med. 2008;132(11):813-820.

6. Krings G, McIntire P, Shin SJ. Myofibroblastic, fibroblastic and myoid lesions of the breast. Semin Diagn Pathol. 2017;34(5):427-437.

7. Salemis NS, Nakos G, Tsiambas E, et al. Rapidly growing myofibroblastoma of the breast diagnosed in a premenopausal woman: management and review of the literature. Breast Dis. 2012;34(1):29-34.

8. Moore T, Lee AH. Expression of CD34 and bcl-2 in phyllodes tumors, fibroadenomas and spindle cell lesions of the breast. Histopathology. 2001;38(1):62-67.

9. Aytaç HÖ, Bolat FA, Canpolat T, et al. Myofibroblastoma of the Breast. $J$ Breast Health. 2015;11(4):192-194.

10. Howitt BE, Fletcher CD. Mammary-type myofibroblastoma: clinicopathologic characterization in a series of 143 cases. Am J Surg Pathol. 2016;40(3):361-367.

11. Shivali B, Chandramouleeswari K, Anita S. Myofibroblastoma breast with unusual morphological features. Cytohistopathogical diagnostic pitfalls and role of immunohistochemistry-review of literature. J Clin Diagn Res. 2013;7(10):2323-2325. 
12. Pauwels P, Sciot R, Croiset F, et al. Myofibroblastoma of the breast: genetic link with spindle cell lipoma. J Pathol. 2000;191(3):282-285.

13. Dal Cin P, Sciot R, Polito P, et al. Lesions of $13 q$ may occur independently of deletion of $16 \mathrm{q}$ in spindle cell/pleomorphic lipomas. Histopathology. 1997;31(3):222-225.

14. Adeniran A, Al-Ahmadie H, Mahoney MC, et al. Granular cell tumor of the breast: a series of 17 cases and review of the literature. Breast $J$. 2004;10(6):528-531.

15. Akahane $\mathrm{K}$, Kato K, Ogiso S, et al. Malignant granular cell tumor of the breast: case report and literature review. Breast Cancer. 2015;22(3):317323
16. Aytac HÖ, Bolat FA, Canpolat T, et al. Myofibroblastoma of the Breast. $J$ Breast Health. 2015;11(4):192-194.

17. Shanmugasiva VV, Ramli Hamid MT, Fadzli F, et al. Myofibroblastoma of the breast. Malays J Pathol. 2018;40(3):349-353.

18. Lee E, Chang YW, Jin YM, et al. Multimodality images of myofibroblastoma in the male breast: A case report and a review of the literature. Clin Imaging. 2018;51:300-306. 